\title{
HOLOMORPHIC QUASIREGULAR MAPPINGS
}

\author{
E. A. POLETSKY
}

\begin{abstract}
Holomorphic quasiregular mappings in bounded domains in $\mathbf{C}^{n}$ are studied. It is shown that the growth of the Jacobian of these mappings depends on the behavior of the boundary of a domain. In particular, the Jacobian is bounded when the boundary is smooth. Some applications to the theory of quasiregular mappings between Hermitian manifolds are given.
\end{abstract}

Introduction. Quasiregular mappings generalize analytic functions on the complex plane to spaces of higher dimension. The definition of quasiregular mappings is related to the concept of conformality, while holomorphic mappings are defined via the Cauchy-Riemann equations.

Both classes of mappings are rich and have a well-developed theory. But it was noted in [1] and proved in [2] in a more general situation that if a mapping $f$ : $\mathbf{C}^{n} \rightarrow \mathbf{C}^{n}$ is simultaneously holomorphic and quasiregular then it is affine. Hence, these notions generalize the concept of analytic functions in different directions. In our paper we support this point of view, proving that the class of holomorphic quasiregular mappings in domains of $\mathbf{C}^{n}, n \geqslant 2$, is very rigid. In particular, these mappings are Lipschitz in domains with a smooth boundary.

The author's interest in this research was aroused after reading [3], where such mappings were used to obtain sharper results in the theory of equidistributions of values, and [4], where it was proposed to study these mappings for such purposes. We show here that in some cases this class is trivial.

Definitions. Let $D$ be a domain in $\mathbf{C}^{n}, n \geqslant 2$, and let $B(z, r)$ be the ball of radius $r$ with center $z, \rho(A, B)$ the Euclidean distance between sets $A$ and $B$. If $z=$ $\left(z_{1}, z_{2}, \ldots, z_{n}\right)$ is a point of $\mathbf{C}^{n}$, then ${ }^{\prime} z=\left(0, z_{2}, \ldots, z_{n}\right),\|z\|^{2}=\sum\left|z_{j}\right|^{2}$ and $e_{1}=$ $(1,0, \ldots, 0)$. For any two real continuous nondecreasing functions $\varphi_{1}$ and $\varphi_{2}$ on $R^{+}$ and $a \in R^{+}$we define a set $A_{1}\left(\varphi_{2}, a\right)$ of points $z=t e_{1}{ }^{+} z$ such that $0<t<a$, $\left\|{ }^{\prime} z\right\|<\varphi_{2}(t)$ and a domain $A\left(\varphi_{1}, \varphi_{2}, a\right)$ consisting of all points $w \in \mathbf{C}^{n}$ such that $\|w-z\|<\varphi_{1}(t)$ for some $z \in A_{1}\left(\varphi_{2}, a\right), z=t e_{1}+{ }^{\prime} z$.

Let $N$ be the complex hyperplane $\left\{z_{1}=0\right\}$.

We shall say that a mapping $f: D \rightarrow \mathbf{C}^{n}$ is $K$-quasiregular if $f \in C^{1}(D)$ and

$$
\left\|f^{\prime}(z)\right\| \leqslant K\left|\operatorname{det} f^{\prime}(z)\right|^{1 / n}, \quad K \geqslant 1,
$$

where $f^{\prime}(z)$ is the derivative of $f$ at the point $z$. Let $J(z)=\operatorname{det} f^{\prime}(z)$.

Received by the editors February 20, 1984 and, in revised form, October 26, 1984. 1980 Mathematics Subject Classification. Primary 32H30. 


\section{Basic lemmas.}

LEMMA 1. Let $f: A\left(\varphi_{1}, \varphi_{2}, a\right) \rightarrow \mathbf{C}^{n}$ be a nonconstant holomorphic quasiregular mapping. Then for any $z \in A_{1}\left(\varphi_{2}, a\right)$ such that $z=t e_{1}+{ }^{\prime} z$,

$$
|\ln J(z)| \leqslant\left|\ln J\left(a e_{1}+{ }^{\prime} z\right)\right|+C \int_{t}^{a} \frac{d \tau}{\varphi_{1}(\tau)}
$$

where $C$ depends only on $K$ and $n$.

Proof. It is well known [2] that, for a holomorphic quasiregular mapping $f$, $J(z)=\operatorname{det} f^{\prime}(z) \neq 0$. Since the domain $A=A\left(\varphi_{1}, \varphi_{2}, a\right)$ is simply connected we can define a function $g(z)=J^{1 / n}(z)$ in $A$ and holomorphic functions

$$
\omega_{k j}=\frac{\partial f_{k}}{\partial z_{j}} / g(z)
$$

where $f_{k}$ is the $k$ th coordinate of $f$.

By (1) and Hadamard's inequality, it follows for the matrix $\Omega=\left(\omega_{k j}\right)$ that

$$
\operatorname{det} \Omega=1 \text { and } 1 \leqslant\|\Omega\| \leqslant K \text {. }
$$

Hence, $\left|\omega_{k j}\right| \leqslant K$ in $A$.

We introduce the bounded holomorphic 1-forms $\omega_{k}=\sum_{j=1}^{n} \omega_{k j} d z_{j}$. Then $d f_{k}=$ $g \omega_{k}$ and $d g \wedge \omega_{k}+g d \omega_{k}=0$ or, equivalently

$$
d \ln g \wedge \omega_{k}=-d \omega_{k} .
$$

If we take the values of both sides of (3) on constant vector fields $x(z) \equiv e_{1}$ and $Y(z) \equiv y \in N$, where $\|y\|=1$, then we obtain that

$$
\frac{\partial \ln g}{\partial X} \omega_{k}(Y)-\frac{\partial \ln g}{\partial Y} \omega_{k}(X)=\frac{\partial \omega_{k}(X)}{\partial Y}-\frac{\partial \omega_{k}(Y)}{\partial X} .
$$

For a point $w=\tau e_{1}+{ }^{\prime} w,\left\|{ }^{\prime} w\right\| \leqslant \varphi_{2}(\tau)$ it follows that the ball $B\left(w, \varphi_{1}(\tau)\right) \subset A$ and, since $\left|\omega_{k}(X)\right|$ and $\left|\omega_{k}(Y)\right|$ are less than or equal to $K$, by Cauchy's inequality, we have

$$
\left|\frac{\partial \omega_{k}(X)}{\partial Y}\right|(w) \leqslant \frac{K}{\varphi_{1}(\tau)}, \quad\left|\frac{\partial \omega_{k}(Y)}{\partial X}(w)\right| \leqslant \frac{K}{\varphi_{1}(\tau)} .
$$

Therefore, at a point $w$,

$$
\left|\frac{\partial \ln g}{\partial X} \omega_{k}(Y)-\frac{\partial \ln g}{\partial Y} \omega_{k}(X)\right| \leqslant \frac{2 K}{\varphi_{1}(\tau)} .
$$

Since the vector $u=\left(\omega_{1}(Y), \ldots, \omega_{n}(Y)\right)$ is equal to $\Omega Y$ and $v=\left(\omega_{1}(X), \ldots, \omega_{n}(X)\right)$ is equal to $\Omega X$, then the inequality (5) yields

$$
\left\|\Omega\left(\frac{\partial \ln g}{\partial X} Y-\frac{\partial \ln g}{\partial Y} x\right)\right\|^{2} \leqslant \frac{4 K^{2} n}{\varphi_{1}^{2}(\tau)} .
$$

Let $\lambda_{1} \leqslant \lambda_{2} \leqslant \cdots \leqslant \lambda_{n}$ be the eigenvalues of $\Omega * \Omega$. By (2), $\operatorname{det} \Omega * \Omega=\Pi \lambda_{k}=1$, and $\lambda_{n}=\|\Omega * \Omega\| \leqslant K^{2}$. Hence,

$$
(\Omega x, \Omega x)=(x, \Omega * \Omega x) \geqslant \lambda_{1}\|x\|^{2} \geqslant\|x\|^{2} / K^{2(n-1)}
$$


and

$$
\begin{aligned}
\left\|\Omega\left(\frac{\partial \ln g}{\partial X} Y-\frac{\partial \ln g}{\partial Y} X\right)\right\|^{2} & \geqslant\left\|\frac{\partial \ln g}{\partial X} Y-\frac{\partial \ln g}{\partial Y} X\right\|^{2} / K^{2(n-1)} \\
& \geqslant\left[\left|\frac{\partial \ln g}{\partial X}\right|^{2}+\left|\frac{\partial \ln g}{\partial Y}\right|^{2}\right] / K^{2(n-1)} .
\end{aligned}
$$

Using the upper estimation (6) for the left side of the last inequality, we see that

$$
\left|\frac{\partial \ln g}{\partial X}\right| \leqslant \frac{2 K^{n} n^{1 / 2}}{\varphi_{1}(\tau)}, \quad\left|\frac{\partial \ln g}{\partial Y}\right| \leqslant \frac{2 K^{n} n^{1 / 2}}{\varphi_{1}(\tau)} .
$$

We consider a segment $I=\left\{w: w=\tau e_{1}+{ }^{\prime} z, t \leqslant \tau \leqslant a,\left\|^{\prime} z\right\| \leqslant \varphi_{2}(t)\right\}$. Since the function $\varphi_{2}$ is nondecreasing, then $I \subset A_{1}\left(\varphi_{2}, a\right)$ and inequalities (7) hold for any point $w \in I$. Therefore,

$$
\left|\ln J(z)-\ln J\left(a e_{1}+{ }^{\prime} z\right)\right|=n\left|\int_{I}\right| \frac{\partial \ln g}{\partial X} d \tau \mid \leqslant C \int_{t}^{a} \frac{d \tau}{\varphi_{1}(\tau)}
$$

and

$$
|\ln J(z)| \leqslant\left|\ln J\left(a e_{1}+{ }^{\prime} z\right)\right|+C \int_{t}^{a} \frac{d \tau}{\varphi_{1}(\tau)} .
$$

Lemma 1 is proved.

The next lemma is trivial.

LEMma 2. Suppose that $h_{j}(t), j=1, \ldots, N$, are smooth complex-valued functions on an interval $I=[0,1]$ such that, at each point $t,\left|h_{j}(t)\right|>a>0$ for some index $j$. Then there is a piecewise-smooth function $h,|h|>a$, and $h(t)=\left|h_{j}(t)\right|$ for some index $j$, and $|h|$ is a continuous function.

Now we can prove a basic

LeMmA 3. Let $f: A\left(\varphi_{1}, \varphi_{2}, a\right) \rightarrow \mathbf{C}^{n}$ be a holomorphic quasiregular mapping. Then for any $z=t e_{1}, 0<t \leqslant a$,

$$
\begin{aligned}
|\ln | J(z)|| \leqslant & C_{1}+|\ln | J\left(a e_{1}\right)|| \\
& +C_{2} \int_{t}^{a} \frac{|\ln | J\left(a e_{1}\right)||}{\varphi_{2}(\tau)} d \tau+C_{4} \int_{t}^{a} \frac{d \tau}{\varphi_{2}(\tau)} \times \int_{\tau}^{a} \frac{d s}{\varphi_{1}(s)},
\end{aligned}
$$

where $C_{i}, i=1, \ldots, 4$, depend only on $a, \varphi_{1}(a), K$ and $n$.

Proof. As in the proof of Lemma 1, we consider constant vector fields $X$ and $Y$. Let $w=\tau e_{1}$ and

$$
\Delta_{\tau}=\left\{z: z=\tau e_{1}+\zeta Y, 0<\tau<a,|\zeta|<\varphi_{2}(\tau)\right\} .
$$

The set $\Delta_{\tau}$ is contained in $A_{1}$, and if $z_{1}=\tau e_{1}+\zeta Y \in \Delta_{\tau}$, then, by Lemma 1,

$$
\left|\ln g\left(z_{1}\right)\right| \leqslant\left|\ln g\left(a e_{1}+\zeta Y\right)\right|+C \int_{\tau}^{a} \frac{d s}{\varphi_{1}(s)}=\psi(\zeta, \tau)
$$


Therefore, by Cauchy's inequality and (7) it follows that

$$
\left|\frac{\partial \ln g}{\partial Y}(w)\right| \leqslant \frac{\psi(0, \tau)}{\varphi_{2}(\tau)}+\frac{C}{\varphi_{1}(a)}=\psi_{1}(\tau)
$$

and $\left|\partial w_{k}(X) / \partial Y\right| \leqslant K / \varphi_{2}(\tau)$.

The last inequalities, together with (4), imply that

$$
\left|\frac{\partial \ln g}{\partial X} w_{k}(Y)+\frac{\partial w_{k}(Y)}{\partial X}\right|(w) \leqslant K \psi_{1}(\tau)+\frac{K}{\varphi_{2}(\tau)}=\psi_{3}(\tau)
$$

or, equivalently,

$$
\left|\frac{\partial \ln \left(g w_{k}(Y)\right)}{\partial X}\right| \leqslant \frac{\psi_{3}(\tau)}{\left|w_{k}(Y)\right|}
$$

if $w_{k}(Y) \neq 0$.

Since $\|\Omega Y\| \geqslant K^{1-n}$, then at any point $w=\tau e_{1}$, for some $k$,

$$
K>\left|w_{k}(Y)\right|>C_{5}=2^{-1} n^{-1 / 2} K^{1-n} .
$$

Hence, there is a function $h,|h|>C_{5}$, defined by Lemma 2 , such that an inequality $|\partial \ln (g h) / \partial X| \leqslant \psi_{3}(\tau) / C_{5}$ holds for $\tau \in[t, a]$ except at a finite number of points. But

$$
\left|\frac{\partial \ln g h}{\partial X}\right| \geqslant\left|\frac{\partial \ln |g h|}{\partial X}\right|
$$

and, since $|g h|$ is a continuous function,

$$
|\ln | g h\left(a e_{1}\right)|-\ln | g h(z)|| \leqslant C_{5}^{-1} \int_{t}^{a} \psi_{3}(\tau) d \tau .
$$

Using the inequality $|h|>C_{5}$ and the expression for $\psi_{3}$, we obtain

$$
\begin{aligned}
|\ln | g(z)|| \leqslant & |\ln | g\left(a e_{1}\right)||+C_{1}^{\prime} \\
& +C_{2}^{\prime} \int_{t}^{a} \frac{|\ln | g\left(a e_{1}\right)||+C_{3}^{\prime}}{\varphi_{2}(\tau)} d \tau+C_{4}^{\prime} \int_{t}^{a} \frac{d \tau}{\varphi_{2}(\tau)} \int_{\tau}^{a} \frac{d s}{\varphi_{2}(s)}
\end{aligned}
$$

where $C_{i}^{\prime}$ depend only on $\varphi_{1}(a), a, K$ and $n$.

Lemma 3 is proved.

Holomorphic quasiregular mappings in $\mathbf{C}^{n}$. Let $\mathfrak{A}\left(\varphi_{1}, \varphi_{2}, a\right)$ be the set of all domains which can be obtained from $A\left(\varphi_{1}, \varphi_{2}, a\right)$ by complex motions. If $G$ is such a domain, we shall denote by $T_{G}$ a complex motion, transforming $A\left(\varphi_{1}, \varphi_{2}, a\right)$ onto $G$. A domain $D \subset \mathbf{C}^{n}$ has type $\left(\varphi_{1}, \varphi_{2}, b, N\right)$ if for any point $z \in D$ there are domains $G_{k} \subset D, G_{k} \in \mathfrak{A}\left(\varphi_{1}, \varphi_{2}, a\right), 1 \leqslant k \leqslant M \leqslant N$, and real numbers $t_{k}, 0<t_{k}$ $\leqslant a$, such that

(1) $T_{G_{1}}\left(t_{1} e_{1}\right)=z$;

(2) $T_{G_{k}}\left(t_{k} e_{k}\right)=T_{G_{k-1}}\left(a e_{1}\right), 2 \leqslant k \leqslant M$;

(3) $\rho\left(T_{G_{M}}\left(a e_{1}\right), \partial D\right) \geqslant b$. 
THEOREM 1. If a domain $D \subset \mathbf{C}^{n}$ has type $\left(\varphi_{1}, \varphi_{2}, b, N\right)$ and $f$ is a holomorphic quasiregular mapping on $D$, then

$$
|\ln | J(z)|| \leqslant P_{N}\left(\int_{t_{k}}^{a} \frac{d \tau}{\varphi_{2}(\tau)}, \quad \int_{t_{k}}^{a} \frac{d \tau}{\varphi_{2}(\tau)} \int_{\tau}^{a} \frac{d s}{\varphi_{1}(s)}\right),
$$

where $P_{N}$ is a polynomial with coefficients, depending only on $K, n, N, \varphi_{1}(a)$ and $C=\sup \{|\ln | J(w)||: \rho(w, \partial D) \geqslant b\}$.

For a proof of Theorem 1 we apply Lemma $3 N$.

COROllary 1. If a domain $D \subset \mathbf{C}^{n}$ has type $\left(c t^{\alpha_{1}}, c t^{\alpha_{2}}, b, N\right)$ where $\alpha_{2}<1$ and $\alpha_{1}+\alpha_{2}<2$, then for any holomorphic quasiregular mapping $f$ on $D,\left\|f^{\prime}\right\|$ is bounded.

Corollary 1 follows from the finiteness of all integrals in the right side of (9).

COROllary 2. If a domain $D \subset \mathbf{C}^{n}$ has a boundary of class $C^{2}$, then for any holomorphic quasiregular mapping $f$ on $D,\left\|f^{\prime}\right\|$ is bounded.

It is easy to see that such a domain has type $\left(c t, c t^{1 / 2}, b, 1\right)$ and, therefore, our statement is a consequence of Corollary 1.

Corollary 3. Let $U=\left\{z \in \mathbf{C}^{n}:\left|z_{i}\right|<1\right\}$ be a polydisk, $E=\left\{z \in U: z_{i}=0, i=\right.$ $1, \ldots, k\}$. Then any holomorphic quasiregular mapping, defined on $U \backslash E$, extends holomorphically to $U$.

If $U_{r}=\left\{z \in \mathbf{C}^{n}:\left|z_{i}\right|<1-r\right\}$, then for any point $z \in U_{2 r}$ we can find a domain $G \in \mathfrak{A}(t, r, r), G \subset U_{r}$, such that $z=T_{G}\left(\left|z_{1}\right| e_{1}\right)$. Hence, by Lemma 3, it follows that $J(z)$ and $\left\|f^{\prime}\right\|$ are bounded in $U_{2 r} \backslash E$ and $f$ extends holomorphically on $E \cap U_{2 r}$. Since it holds for all such $r$, we proved the corollary.

EXAmple 1. Let $D=\left\{x \in \mathbf{C}^{2}: K^{-1}<\left|z_{1}\right| /\left|z_{2}\right|<K,\left|z_{1}\right|<1\right\}$ and $f=\left(z_{1}^{-n}, z_{2}^{-n}\right)$. It is easy to see that $D$ has type ( $c t, c t, b, 2), f$ is holomorphic and quasiregular, but $J(z)=n^{2}\left(z, z_{2}\right)^{-(n+1)}$ is unbounded. This example shows that our estimates in Corollary 1 are best possible.

EXAMPLE 2. One might hope that there are nontrivial estimates for higher derivatives of a holomorphic quasiregular mapping. The next example shows that this hope is in vain.

Let $D=B(0,1) \subset C^{2}$ and $f=\left(z_{1}+\left(z_{1}-1\right)^{3 / 2} / 3, z_{2}\right)$. By a direct calculation it is easy to check that $J(z) \geqslant 0,25,\left\|f^{\prime}\right\| \leqslant 5$. Hence, $f$ is quasiregular but the second derivative of $f$ is unbounded.

Holomorphic quasiregular mappings between manifolds. A holomorphic mapping $f:(M, g) \rightarrow(N, h)$ of two Hermitian manifolds $M$ and $N$ of the same dimension with Hermitian metrics $g$ and $h$ is called quasiregular if its derivative $f^{\prime}$ satisfies an inequality (1) at any point $w \in M$. In this case, we must calculate $\left\|f^{\prime}\right\|$ and det $f^{\prime}$ with respect to the metrics $g$ and $h$. Two metrics $g_{1}$ and $g_{2}$ on $M$ are quasiconformally equivalent if the identity mapping $i:\left(M, g_{1}\right) \rightarrow\left(M, g_{2}\right)$ is quasiregular. In this case, if a mapping $f:\left(M, g_{1}\right) \rightarrow(N, h)$ is quasiregular, then $f:\left(M, g_{2}\right) \rightarrow(N, h)$ is quasiregular too. 
THEOREM 2. Let $A$ be a compact complex Hermitian manifold with metric $g$ and let $B$ be its analytic subvariety. Then any quasiregular holomorphic mapping $f:(A \backslash B, g) \rightarrow$ $\mathrm{C}^{n}$ is constant.

Proof. Let $B_{1}$ be the set of singular points of $B$. It is well known that $\operatorname{dim} B_{1}<\operatorname{dim} B$. We choose new coordinates $z_{1}, \ldots, z_{n}$ in some neighborhood $V$ of a point $z \in B \backslash B_{1}$ such that $B \cap V=\left\{w \in V: w_{i}=0, i=1, \ldots, k\right\}$. The mapping $f$ is quasiregular with respect to the Euclidean metric on $V$ and, hence, by Corollary 3, extends holomorphically to $B \cap V$. Since $z$ is any point of $B \backslash B_{1}, f$ extends to $B \backslash B_{1}$ and, therefore, can be defined on $A \backslash B_{1}$. Repeating this procedure if necessary, we can extend $f$ to $A$. But $A$ is compact and, hence, $f$ is constant.

THeOREM 3. Let $B$ be a subvariety of $\mathbf{C}^{n}, n>1$. Then any quasiregular holomorphic mapping $f: \mathbf{C}^{n} \backslash B \rightarrow \mathbf{C}^{n}$ is affine.

Proof. As in the proof of Theorem 2, $f$ extends to $\mathbf{C}^{n}$ and our theorem follows by a result in $[1,2]$ that any quasiregular holomorphic mapping $f: \mathbf{C}^{n} \rightarrow \mathbf{C}^{n}$ is affine.

The next theorem gives an answer to Problem 14 in [3].

THEOREM 4. Let $\mathbf{C} P^{n}$ be the complex projective space with Fubini-Study metric. Then any holomorphic quasiregular mapping $f: \mathbf{C}^{n} \rightarrow \mathbf{C} P^{n}$ is constant.

Proof. Let $g$ be a Fubini-Study metric, $\phi$ the fundamental 2 -form and $\rho$ its Ricci form. In local coordinates, $\rho=i \partial \bar{\partial} \log G$ where $G$ is the determinant of the matrix, corresponding to the quadratic form $g$. It can be checked by a straightforward calculation that for any holomorphic vector field $X$ the form

$$
\Omega=(i / 2) \partial \bar{\partial} \log \phi(X, i X)-\rho / 2 n
$$

is positive.

We shall denote by an asterisk the pull-back by $f$ of a corresponding object (and suppose that $f$ is nonconstant). Then the form

$$
\Omega_{1}=(i / 2) \partial \bar{\partial} \log \phi^{*}(X, i X)-i \partial \bar{\partial} \log G^{*} / 2 n
$$

is positive too, and this means that the function

$$
h_{X}(z)=\log \frac{\phi^{*}(X, i X)}{\left(G^{*}\right)^{1 / n}}(z)
$$

is plurisubharmonic. By the quasiregularity of $f$, it follows that

$$
K^{-1}\|X\|^{2} \cdot\left(G^{*}\right)^{1 / n} \leqslant \phi^{*}(X, i X) \leqslant K\|X\|^{2}\left(G^{*}\right)^{1 / n} .
$$

Hence, for any constant vector field $X$ on $\mathbf{C}^{n}$, the function $h_{X}$ is constant, because any bounded plurisubharmonic function in $\mathbf{C}^{n}$ is constant, and this means that the form $\phi_{1}=\phi^{*}\left(G^{*}\right)^{-1 / n}$ has constant coefficients. But $g$ is a Kaehler metric. Therefore, $d \phi=d \phi^{*}=0$ and

$$
d \phi_{1}=\phi^{*} \wedge d\left(G^{*}\right)^{-1 / n}=0 .
$$

Since $\phi^{*}$ is positive, $d G^{*}=0$ and $G^{*} \equiv$ const which contradicts the positiveness of the Ricci form.

The theorem is proved. 


\section{REFERENCES}

1. O. V. Titov, Quasiconformal harmonic mappings of Euclidean space, Dokl. Akad. Nauk SSSR 194 (1970).

2. A. Marden and S. Rickman, Holomorphic mappings of bounded distortion, Proc. Amer. Math. Soc. 46 (1974), 225-228.

3. H. Wu, Remarks on the first main theorem in equidistribution theory. IV, J. Differential Geom. 2 (1969), 433-446.

4. P. Griffiths, Holomorphic mappings into canonical algebraic varieties, Ann. of Math. (2) 93 (1971), 439-458.

Moscow, USSR 